Meta

Journal des traducteurs

Translators' Journal

\title{
Peux-tu me donner un « lift »?
}

\section{Jeanette Oundjian-Crosby et Colette Joy}

Volume 25, numéro 3, septembre 1980

URI : https://id.erudit.org/iderudit/002439ar

DOI : https://doi.org/10.7202/002439ar

Aller au sommaire du numéro

Éditeur(s)

Les Presses de l'Université de Montréal

ISSN

0026-0452 (imprimé)

1492-1421 (numérique)

Découvrir la revue

Citer cet article

Oundjian-Crosby, J. \& Joy, C. (1980). Peux-tu me donner un « lift »? Meta, 25(3), 362-364. https://doi.org/10.7202/002439ar d'utilisation que vous pouvez consulter en ligne.

https://apropos.erudit.org/fr/usagers/politique-dutilisation/ 


\section{PEUX-TU ME DONNER UN «LIFT »}

Le mot lift est un emprunt qui figure dans bon nombre de locutions de la langue populaire du Canada Français. C'est un anglicisme employé couram-

24. McGraw-Hill Encyclopedia of Science and Technology, New York, McGraw-Hill Book Company, 1977 , Vol. 5 , p. 16

25. Webster's Third New International Dictionary with Seven Language Dictionary, op. cit., sv.

26. STOP (Society to Overcome Pollution), Bruce Walker, Research Coordinator.

Note: The Encyclopedia Americana, Vol. 7, p. 486, contains a glossary of commonly used environmental terms, with descriptive detail. 
ment dans des expressions telles que «Peux-tu me donner un lift jusqu'à l'université ?», «Pierre m'a donné un lift », «J'ai eu un lift pour venir».

Lift signifie «A ride along one's way in a vehicle going in the same direction. Ex.: He gave her lifts in his car between there and the village. Couple raising their thumbs for a lift) ${ }^{1}$.

Le mot ride recouvre dans ce contexte l'idée de transport gratuit. Habituellement, le trajet que parcourt l'automobiliste est le même que celui du piéton, mais ce n'est pas toujours le cas. La personne que fait monter l'automobiliste peut être une connaissance ou un étranger (un auto-stoppeur, par exemple). Le terme lift implique la notion de "bienveillance» de la part de l'automobiliste à l'égard de la personne qu'il fait monter dans son véhicule.

Il n'existe pas d'équivalent universel qu'on pourrait infailliblement substituer à cet anglicisme. Des tructures telles que «J'ai donné...» ou «Peux-tu me donner...» appellent inconsciemment le mot lift. Pour rendre cette notion dans un français idiomatique, il faut employer la tournure qui surgirait spontanément à l'esprit d'un francophone placé dans la même situation de communication.

G. Colpron ${ }^{2}$ et G. Dagenais ${ }^{3}$ recommandent l'emploi du mot «occasion» pour rendre lift employé dans le sens de «transport gratuit par bienveillance»:

- J'ai eu une occasion ce matin, c'est pourquoi j'arrive plus tôt que d'habitude.

- Nous avons eu deux occasions en nous rendant de Québec à Montréal.

- Avoir une occasion pour se rendre en ville. Etc.

«Le mot occasion, précise Dagenais, ne peut s'employer, cependant, pour désigner le fait de faire monter quelqu'un dans sa voiture et de le transporter gratuitement. On peut dire «J'ai fourni à un jeune homme 1'occasion de se rendre gratuitement de... à...», mais c'est du langage écrit plutôt que du language parlé. On dira plus familièrement «J'ai transporté un jeune auto-stoppeur de... à...» ou «J'ai pris, au sens d'accueillir, un auto-stoppeur», etc.

Or, il ne faut pas essayer de faire correspondre à tout prix une unité lexicale d'une langue à une autre de la langue d'arrivée, car on risque de faire violence à l'idiomatique de cette langue. En effet, les équivalences de traduction ne sont pas toujours lexicales; elles peuvent aussi être contextuelles ou périphrastiques. Ainsi, même s'il n'y a pas de mot passe-partout pour remplacer lift, il existe bien des tournures françaises syntagmatiques pour combler cette lacune. On ne traduit pas la langue, mais des messages.

On peut citer, à titre d'exemples, les possibilités suivantes :

- Veux-tu monter avec moi?

- Puis-je vous reconduire?... vous déposer chez vous ${ }^{4}$ ?

1. Webster's Third International Dictionary of the English Language, 1971.

2. Gilles Colpron, Les Anglicismes au Québec, Montréal, Librairie Beauchemin Limitée, 1977.

3. Gérard Dagenais, Dictionnaire des difficultés de la langue française au Canada, QuébecMontréal, Editions Pedagogia Inc., 1967.

4. Fiche de Radio-Canada, 1969. 
- Est-ce que je peux vous déposer quelque part?

- Je l'ai pris en voiture.

- Je l'ai emmené jusqu'à Paris ${ }^{5}$.

- Voulez-vous profiter de ma voiture?

- Je vais vous conduire un bout ${ }^{6}$.

Il est intéressant de noter ici que seules les sources canadiennes proposent l'emploi du terme «occasion».

En somme, cette courte étude de la traduction du mot lift illustre un principe important de la traduction: les équivalences interlinguistiques ne sont pas toujours lexicalement possibles. Toutes les langues peuvent tout dire, tout exprimer. C'est la façon de le faire qui diffère d'une langue à une autre.

«Languages, a écrit Roman Jakobson, differ essentially in what they must convey and not in what they may convey ${ }^{7}$.» L'équivalence dans la différence est le problème cardinal de la traduction. Lift en fournit un bon exemple.

JEANNETTE OUNDJIAN-Crosby

COLETTE JoY 